\title{
Model-Based Throttle Control using Static Compensators and Pole Placement
}

\author{
A. Thomasson* and L. Eriksson \\ Vehicular Systems, Dept. of Electrical Engineering, Linköping University, 58183 Linköping - Sweden \\ e-mail: andreasł@isy.liu.se - larer@isy.liu.se \\ * Corresponding author
}

\begin{abstract}
Résumé - Commande des gaz basée sur un modèle utilisant des compensateurs statiques et un placement de pôles - Au sein des moteurs à allumage commandé modernes, les gaz sont régulés par le boîtier de commande électronique (ECU; Electronic Control Unit), qui permet la régulation directe par l'ECU du flux d'air et ainsi du couple moteur. Cela conduit à des exigences élevées quant à la vitesse et à la précision du régulateur qui positionne le papillon des gaz. Le problème de commande des gaz est compliqué par deux forts effets non linéaires, le frottement et le couple de mode de secours ("limp-home"). Cet article propose l'utilisation de deux compensateurs statiques, actifs simultanément, pour contrer ces effets et linéariser approximativement le système. Un régulateur PID est conçu pour le système linéarisé, où un placement de pôles est appliqué pour concevoir le régulateur PD et une partie I à gains paramétrés est ajoutée pour la robustesse par rapport aux erreurs de modèle. Un mode opératoire systématique destiné à générer les paramètres du compensateur et du régulateur à partir d'expériences en boucle ouverte est également développé. Les performances du régulateur sont évaluées à la fois par simulation, sur un problème de référence de commande des gaz, et expérimentalement. Une étude de robustesse a montré que la position de mode de secours constitue un paramètre important pour les performances du régulateur, ceci étant souligné par les écarts trouvés au cours des expériences. La méthode proposée pour l'identification des paramètres atteint la précision désirée.
\end{abstract}

\footnotetext{
Abstract - Model-Based Throttle Control using Static Compensators and Pole Placement - In modern spark ignited engines, the throttle is controlled by the Electronic Control Unit (ECU), which gives the ECU direct control of the air flow and thereby the engine torque. This puts high demands on the speed and accuracy of the controller that positions the throttle plate. The throttle control problem is complicated by two strong nonlinear effects, friction and limp-home torque. This paper proposes the use of two, simultaneously active, static compensators to counter these effects and approximately linearize the system. A PID controller is designed for the linearized system, where pole placement is applied to design the PD controller and a gain scheduled I-part is added for robustness against model errors. A systematic procedure for generating compensator and controller parameters from open loop experiments is also developed. The controller performance is evaluated both in simulation, on a throttle control benchmark problem, and experimentally. A robustness investigation pointed out that the limp-home position is an important parameter for the controller performance, this is emphasized by the deviations found in experiments. The proposed method for parameter identification achieves the desired accuracy.
} 


\section{INTRODUCTION}

An electronic throttle is a DC-servo that controls the throttle plate in modern Spark Ignited (SI) engines. The position of the throttle plate controls the air-flow to the engine and hence the engine torque. As a consequence this servo is a very important component in a vehicle since it affects the vehicle driveability.

Throttle control design is challenging due to two nonlinearities, friction and limp-home torque, which effects the throttle plate motion. Strategies for overcoming these difficulties have been addressed in several papers. A model based friction compensator was presented in Eriksson and Nielsen (2000) and a nonlinear control strategy with both friction and limp-home compensation is proposed in Deur et al. (2004). Another approach was made in Vašak et al. (2006), where a control law based on the solution of an optimal control problem was demonstrated.

This paper makes use of friction and limp-home compensators that are static functions of the measured throttle position and reference value, to remove most non-linearities. This is a combination of ideas presented in Eriksson and Nielsen (2000) and Deur et al. (2004). The proposed controller uses a Coulomb friction model, which is less complex than the friction models usually proposed in the literature, and a PID-controller with fewer degrees of freedom. This gives fewer parameters which simplifies the tuning procedure. With these simplifications the controller fulfills the requirements in the TC benchmark problem in Zito et al. (2009), similar to those in Deur et al. (2004).

A systematic tuning procedure is described that utilize ramp and step responses in control signal. The PDcontroller is tuned using pole placement, to give a first order behavior with a desired rise time to the linearized system. A robustness investigation is made to analyze the sensitivity to parameter variations. This shows that an accurate estimation of the limp-home position is needed, and a calibration procedure to assure this is suggested.

In the next section a simplified model for an electronic throttle is presented with the aim of controller design. Section 2 describes the controller structure and its three main parts: friction compensator, limp-home compensator and PID-controller. In Section 3, a procedure for identifying the controller parameters are discussed. The controller performance and robustness to parameter variations is verified in simulation on the benchmark model in Section 4, and experimentally on a throttle in Section 5.

\section{CONTROL ORIENTED THROTTLE MODEL}

In this section the throttle model that is used to design the controller in Section 2 is presented. Figure 1 shows a sketch of the throttle system. The control signal is transformed to a PWM signal by the chopper that is connected to the DC

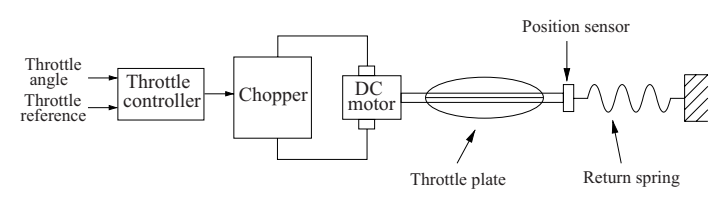

Figure 1

A schematic of the electronic throttle and controller. Main parts are the controller (which is part of the ECU), chopper, DC motor, throttle plate, return spring and position sensor.

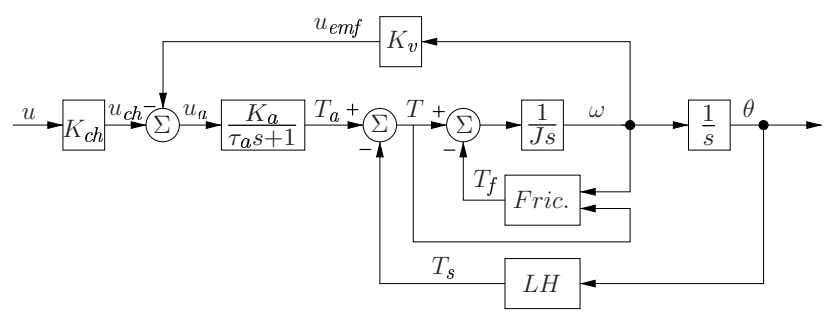

Figure 2

Process model for the electronic throttle. The torque contributions on the throttle plate are the armature torque, $T_{a}$, the friction torque, $T_{f}$, and the spring torque, $T_{s}$. The armature is modeled by a first order system with gain $K_{a}$ and time constant $\tau_{a}$. The chopper and back electromotive torque are modeled with the proportional gains, $K_{c h}$ and $K_{v}$, from the control signal and throttle plate angular velocity, $u$ and $\omega$, respectively.

motor. The motor torque is transferred to the throttle plate axle through a gearbox (not shown in figure). The return spring exercises a torque on the throttle plate that pulls it toward the limp-home position.

A process model for the electronic throttle body is shown in Figure 2, see Scattolini et al. (1997), Deur et al. (2004). The model is a standard linear electric dc-motor, augmented with friction and limp-home torque components. The chopper is modeled with a proportional gain. The armature time constant, $\tau_{a}$, is typically very small, approximately $1 \mathrm{~ms}$ (Deur et al., 2004). This effect is therefore neglected for the purpose of controller design. The armature torque can then be divided in

$$
T_{a}=K_{a} K_{c h} u-K_{a} K_{v} \omega=T_{u}-T_{e m f}
$$

The torque acting on the throttle plate is thus composed of four main parts. The driving torque from the DC motor, $T_{u}$, the spring torque, $T_{s}$, the friction torque, $T_{f}$, and the back electromotive torque, $T_{e m f}$. The friction torque is further divided into a static and dynamic part, $T_{f s}$ and $T_{f v}$. After modeling the separate torque contributions, the equations of motion for the throttle plate are given by Newtons second law.

$$
\begin{aligned}
\dot{\theta} & =\omega \\
J \dot{\omega} & =T_{u}-T_{s}-T_{f s}-T_{f v}-T_{e m f}
\end{aligned}
$$




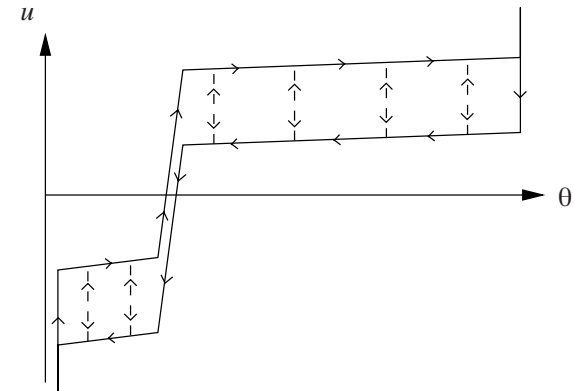

Figure 3

Sketch of the static nonlinearities for the electronic throttle. Arrows indicate the direction of movement. The influence of friction and the nonlinear spring torque is clearly seen. Compare with the measured curve shown in Figure 8.

Both the static friction and limp-home nonlinearity can be seen from the process static curve illustrated in Figure 3. This curve can be estimated by performing a slow ramp in the control signal while measuring the response in throttle position. The influence of friction is clearly seen from the difference in the ramp up and down in control signal. The static friction is modeled using the classical Coulomb friction model (2), Olsson et al. (1998). The friction torque is equal to the applied torque, $T$, when $\omega=0$ and the applied torque is less than the Coulomb friction, $T_{c}$. Otherwise, the friction torque is equal to Coulomb friction in the opposite direction of motion.

$$
T_{f s}(T, \omega)=\left\{\begin{array}{cl}
T & \text { if } \omega=0 \text { and }|T|<T_{c} \\
T_{c} \operatorname{sgn}(\omega) & \text { otherwise }
\end{array}\right.
$$

Several papers suggest the use of more complex, dynamic friction models, and to include Stribeck effect. The Coulomb friction model is chosen because it is simpler and has fewer parameters, whilst it proved to be sufficient for the controller design to meet all benchmark requirements in Zito et al. (2009), similar to those in Deur et al. (2004).

The limp-home nonlinearity comes from the springs that pull the throttle plate toward the limp-home position. The spring torque is piecewise linear but the spring constant differs greatly, depending on whether the throttle plate is inside or outside of the limp-home region. The slope of the $u(\theta)$ curve is almost flat above and below the limp-home region, with a very sharp transition between them. There is approximately $30 \%$ increase in the control signal from fully closed to fully open throttle, where about $20 \%$ is in a narrow region of $0.5-2^{\circ}$ around the limp-home position. The spring torque is therefore described as the piecewise linear function in the equation below and is illustrated in Figure 4.

$$
T_{s}(\theta)= \begin{cases}m_{l h}^{+}+k^{+}\left(\theta-\theta_{l h}^{+}\right) & \text {if } \theta>\theta_{l h}^{+} \\ m_{l h}^{+}\left(\theta-\theta_{l h}\right) /\left(\theta_{l h}^{+}-\theta_{l h}\right) & \text { if } \theta_{l h}<\theta \leq \theta_{l h}^{+} \\ m_{l h}^{-}\left(\theta_{l h}-\theta\right) /\left(\theta_{l h}-\theta_{l h}^{-}\right) & \text {if } \theta_{l h}^{-}<\theta \leq \theta_{l h} \\ m_{l h}^{-}-k^{-}\left(\theta_{l h}^{-}-\theta\right) & \text { if } \theta \leq \theta_{l h}^{-}\end{cases}
$$

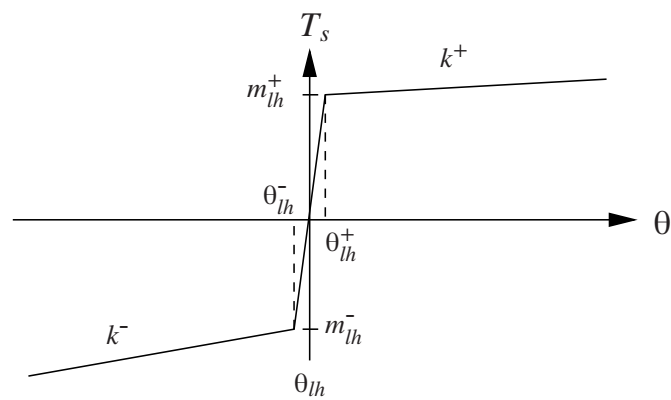

Figure 4

A graphical representation of the model for the spring torque, which is a piecewise linear function of the throttle position.

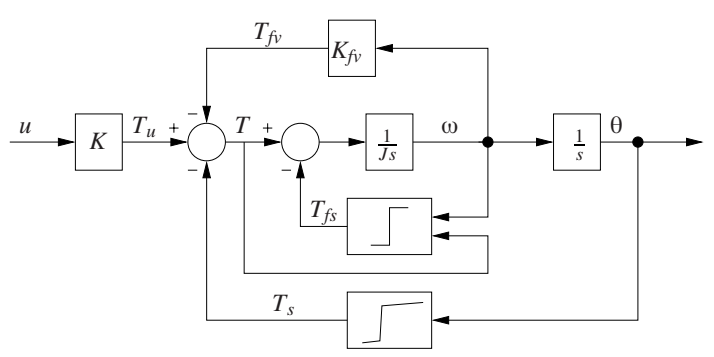

Figure 5

A block diagram showing the simplified process model. The model captures the most important throttle dynamics and is the basis for the controller structure.

The models for viscous friction and electromotive torque are both linear functions in angular velocity acting in the opposite direction of motion. They are lumped into a single torque model.

$$
T_{f v}+T_{e m f}=K_{f v} \omega
$$

Combining Equations (1-3) and (4), gives the differential equation for the throttle plate angular velocity.

$$
J \dot{\omega}=-K_{f v} \omega-T_{s}(\theta)-T_{f s}(T, \omega)+K u
$$

The complete model is shown as a block diagram in Figure 5. This model captures most essential parts of the throttle dynamics and form the basis for the controller structure.

\section{CONTROLLER STRUCTURE}

To linearize the system in Equation (5), a nonlinear compensator block that modifies the control signal $u$ is proposed. The main idea is to choose the control signal as:

$$
u=\frac{T_{s}(\theta)}{K}+\frac{T_{f s}(T, \omega)}{K}+\tilde{u}
$$

which would be an exact linearization of Equation (5). This is not possible due to several reasons, but can be done 
approximately by the compensator blocks described in the two following sections. The linearized system is then controlled by a slightly modified PID-controller described in Sections 2.4 and 2.5 .

\subsection{Limp-Home Compensator}

To exactly counter the limp-home torque this block would use Equation (3) directly. Small variations in the measured output close to the limp-home position would then result in severe chattering of the control signal due to the large slope in the static curve in this region. To overcome this, the commanded throttle reference, $\theta_{\text {ref }}$, is used as input to Equation (3) instead of the measured position. As long as the reference itself does not vary rapidly around the limphome position this will prevent the limp-home compensator from causing chattering in the control. Effectively this is a feedforward with the inverse static gain of the system as output.

\subsection{Friction Compensator}

Based in Equation (2) the compensator would be an ideal relay that switches sign around $\omega=0$. This creates a problem with estimating the speed and direction of the throttle plate motion based on the position measurements. It is also not beneficial if the throttle plate currently is moving away from the reference value and the friction compensator add to that motion. Instead the friction compensator is based directly on the tracking error and compensation is made in the direction that reduces the tracking error. An ideal relay function would be very sensitive to noise around $e_{\theta}=0$ and would also cause undesirable oscillations around the reference value. This problem is solved with a small dead zone around the reference value and a smooth transition when $e_{\theta}$ increases. The dead zone radius is denoted $\theta_{d}$ and the width of the transition $\theta_{r}$, see Figure 6 and Equation (7).

$$
\tilde{T}_{f}\left(e_{\theta}\right)= \begin{cases}0 & \text { if }\left|e_{\theta}\right| \leq \theta_{d} \\ \tilde{T}_{c} \frac{\theta-\theta_{d}}{\theta_{r}} \operatorname{sgn}\left(e_{\theta}\right) & \text { if } \theta_{d}<\left|e_{\theta}\right| \leq \theta_{r}+\theta_{d} \\ \tilde{T}_{c} \operatorname{sgn}\left(e_{\theta}\right) & \text { if }\left|e_{\theta}\right|>\theta_{r}+\theta_{d}\end{cases}
$$

When the throttle plate is close to the reference value a large part of the control signal comes from the friction compensator block. If the Coulomb friction were underestimated the rise time could get unnecessary long after small changes in reference position. To make sure that the friction compensator overcomes the Coulomb friction the maximum amplitude of the compensator block is increased to $\tilde{T}_{c}=T_{c} \times k$, where $k$ is slightly larger than one. Also the Coulomb friction is different above and below the limp-home position and its values are distinguished by $T_{c}^{+}$and $T_{c}^{-}$respectively.

The limp-home and friction compensators are used to modify the control signal according to Equation (8), where $\tilde{u}$ is the output from the PID-controller described next.

$$
u=\frac{T_{s}\left(\theta_{r e f}\right)}{K}+\frac{\tilde{T}_{f}\left(e_{\theta}\right)}{K}+\tilde{u}
$$

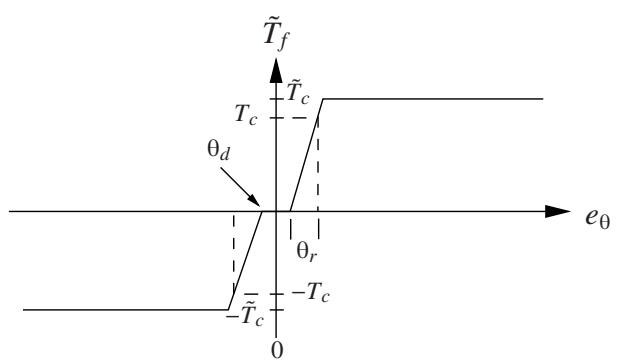

Figure 6

The friction compensation is implemented as a static function of the tracking error. A small dead zone and a smooth transition is used around $e_{\theta}=0$ to make the compensation less sensitive and avoid oscillations close to the reference value.

\subsection{The Linearized System}

With the control signal selected according to Equation (8) and the compensators in Section 2.1 and 2.2, the system is approximately linear and given by the equation:

$$
J \dot{\omega}=-K_{f v} \omega+K \tilde{u}
$$

To simplify notation the equation is normalized by $K_{f v}$ and the parameters $T_{0}=\frac{J}{K_{f v}}$ and $K_{0}=\frac{K}{K_{f v}}$ are introduced. The resulting differential equation is:

$$
T_{0} \dot{\omega}=-\omega+K_{0} \tilde{u}
$$

and the resulting transfer function from $\tilde{u}$ to $\theta$ is:

$$
\theta(s)=\frac{K_{0}}{s\left(T_{0} s+1\right)} \tilde{u}(s)
$$

The throttle position is normalized to $[0,100]$ and the control signal is normalized to $[-100,100]$.

\subsection{PID Design Using Pole Placement}

To avoid hitting the mechanical stops at the end positions, the response to a reference step should have no overshoot. The parameter $T_{0}$ in Equation (11) is typically small, in the order of a few samples of the controller, and the system is simplified to:

$$
\theta(s)=\frac{K_{0}}{s} \tilde{u}(s)=G(s) \tilde{u}(s)
$$

with $G(s)=K_{0} / s$ for synthesis. To avoid overshoot we desire the closed loop system to have a first order behavior, and use pole placement to design the controller (Rivera et al., 1986; Aström and Tore Hägglund, 2006). For the system in Equation (12) this results in a P-controller. The the closed loop system is given by:

$$
G_{c}(s)=\frac{1}{\frac{1}{K_{p} K_{0}} s+1}=\frac{1}{\lambda s+1}
$$


with $K_{p}=\frac{1}{K_{0} \lambda}$, where the tuning parameter $\lambda$ is the desired rise time. This rise time will of course only be achieved when the control signal does not saturate. If the product $K_{0} \cdot \lambda$ is smaller than about one this will unfortunately be the case for larger reference steps.

Using only a P-controller have however shown to give overshoots, most likely due to the approximate linearization and model uncertainties. A derivative part is included in the feedback loop to overcome this. The controller equation and corresponding closed loop system becomes:

$$
\begin{aligned}
\tilde{u}(s) & =K_{p}\left(\theta_{\text {ref }}(s)-\theta(s)\right)-K_{d} s \theta(s) \\
G_{c}(s) & =\frac{1}{\frac{1+K_{d} K_{0}}{K_{p} K_{0}} s+1}=\frac{1}{\lambda s+1} \\
\lambda & =\frac{1+K_{d} K_{0}}{K_{p} K_{0}}
\end{aligned}
$$

This is the same closed loop system as with only a Pcontroller, but with an extra degree of freedom that can be used to adjust the trade-off between the sensitivity function, $S$, and complementary sensitivity function, $T$, according to:

$$
\begin{aligned}
& S(s)=\frac{\frac{1}{K_{p} K_{0}} s}{\frac{1+K_{d} K_{0}}{K_{p} K_{0}} s+1}=\frac{\frac{1}{K_{p} K_{0}} s}{\lambda s+1} \\
& T(s)=\frac{\frac{K_{d}}{K p} s+1}{\frac{1+K_{d} K_{0}}{K_{p} K_{0}} s+1}=\frac{\frac{K_{d}}{K p} s+1}{\lambda s+1}
\end{aligned}
$$

For a given desired rise time $\lambda$, increasing $K_{d}$ will increase $K_{p}$ due to Equation (14c), which will decrease the sensitivity function. At the same time the complementary sensitivity function will increase since $S+T=1$. The strategy for tuning the PD-controller is discussed in Section 3.2.

In the control system the position derivative in Equation (14a) has to be estimated from discrete measurements. To reduce the sensitivity to measurement noise the derivative is approximated with the filtered difference:

$$
D_{y}(z)=\frac{(1-\gamma)\left(1-z^{-1}\right)}{1-\gamma z^{-1}} Y(z)
$$

where $D_{y}(z)$ is the derivative approximation and $Y(z)$ is the measured output. The filter coefficient, $\gamma$, has to be chosen to give the filter a cut-off frequency that is above the frequency range where a reduction in the sensitivity function is desired.

\subsection{A Modified I-Part}

A prerequisite to get the closed loop system behavior in Equation (13) is that the model is correct. Even with a correct model the approximations in the linearization or input disturbances etc. could result in a stationary tracking error. To compensate for this an I-part is added to the controller.

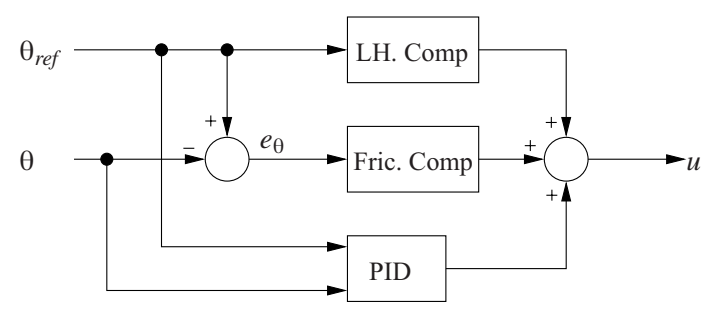

Figure 7

A block diagram of the controller structure. The control signal $u$ is the sum of the outputs from the compensator blocks and the PID-controller.

The integrator gain $K_{i}$ is gain scheduled. It is small when the error is large and increases with decreasing error. This helps to quickly overcome model errors for small changes in reference and prevent overshoot for relatively small steps, that could otherwise result from the large integrator gain.

To prevent wind-up the integrator is turned off when the control signal saturates. One reset condition is also used, that is for reference steps larger than $0.5 \%$. This was introduced because a nonzero integrator before a small reference step, where the control signal does not saturate, was causing overshoot. For this controller, with a sampling rate of $1 \mathrm{~ms}$, a ramp in reference with a change of $0.5 \%$ per sample corresponds to a change from fully closed to fully open throttle in $0.2 \mathrm{~s}$, which is on the edge of what is possible to achieve in a step (see Fig. 11). The integrator would not improve tracking for this rapid change in reference, implying that the reset condition will not have a negative effect on tracking performance. For a lower sampling rate this threshold would have to be increased accordingly. Other standard anti-windup schemes, e.g. tracking Aström and Tore Hägglund (2006), where tried for this controller with slightly worse result, but could be an alternative.

The error input to the integrator is also modified. When the tracking error is smaller than half the resolution of the position measurement, the error is set to zero. A block diagram of the implemented controller structure is shown in Figure 7.

\section{IDENTIFICATION AND CONTROLLER TUNING}

In order to be able to automate the controller parametrization, two experiments are proposed. The experiments are designed to identify the model parameters that directly give the controller parameters in the model-based controller. Both compensator blocks are identified from a ramp response in control signal described in Section 3.1, while the PD-parameters are determined using open and closed loop step responses, described in Section 3.2. 


\subsection{The Static Compensators - Ramp Response}

Both the friction and limp-home nonlinearities are estimated from the process static curve that was illustrated in Figure 3. This characteristic can be measured by doing a slow ramp response up and down in the throttle control signal, see Figure 8. The proposed method for calculating the limphome and friction compensators identifies the points $A_{1}-A_{4}$ and $B_{1}-B_{4}$ marked in the figure by fitting a piecewise linear function to each ramp.

The coulomb friction in the friction compensator block is estimated as half the distance between the two curves. The values above and below the limp-home position are calculated as:

$$
\begin{aligned}
T_{c}^{-} & =\frac{u\left(A_{1}\right)-u\left(B_{1}\right)+u\left(A_{2}\right)-u\left(B_{2}\right)}{4} \\
T_{c}^{+} & =\frac{u\left(A_{3}\right)-u\left(B_{3}\right)+u\left(A_{4}\right)-u\left(B_{4}\right)}{4}
\end{aligned}
$$

The dead zone and transition in the friction compensator should be made small to get precise control for small changes in reference. Making them too small however can cause oscillations around the reference value. In simulation and experimentally $\theta_{d} \approx 0.1 \%$ and $\theta_{r} \approx 0.5 \%$ have proved to work satisfactory.

The equations for calculating the parameters in the limphome compensator are given in Equation (18). For this specific throttle the slope below the limp home position is only slightly higher than the slope above, but this difference can

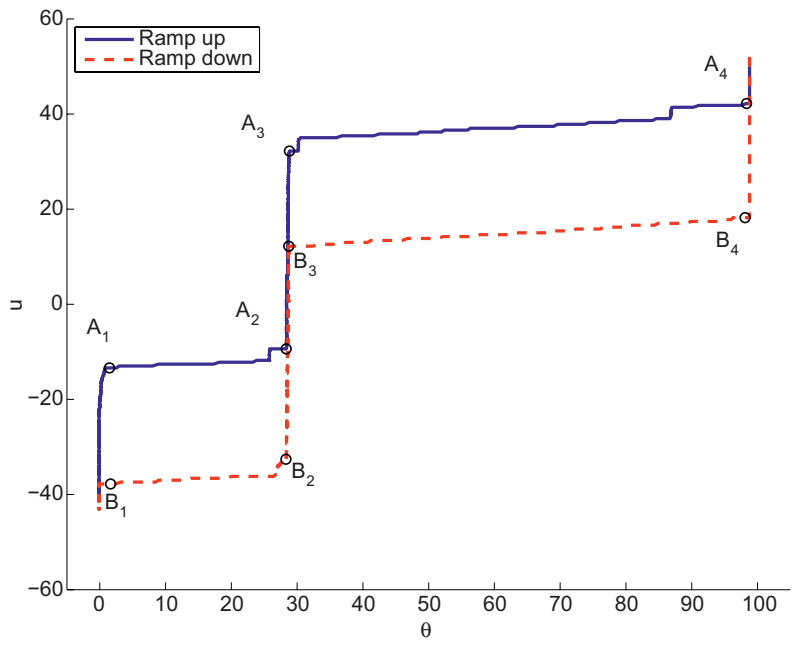

Figure 8

Measured ramp response in throttle position, the solid line (blue) is when $u$ is increasing and the dashed line (red) when $u$ is decreasing. The marked points $A_{1}-A_{4}$ and $B_{1}-B_{4}$ are used in the calibration procedure when calculating the friction and limp-home compensators. be larger, see for example Scattolini et al. (1997), Pavkvić et al. (2006).

$$
\begin{aligned}
\theta_{l h} & =\frac{\theta\left(A_{2}\right)+\theta\left(A_{3}\right)+\theta\left(B_{2}\right)+\theta\left(B_{3}\right)}{4} \\
\theta_{l h}^{-} & =\frac{\theta\left(A_{2}\right)+\theta\left(B_{2}\right)}{2} \\
\theta_{l h}^{+} & =\frac{\theta\left(A_{3}\right)+\theta\left(B_{3}\right)}{2} \\
m_{l h}^{-} & =\frac{u\left(A_{2}\right)+u\left(B_{2}\right)}{2} \\
m_{l h}^{+} & =\frac{u\left(A_{3}\right)+u\left(B_{3}\right)}{2} \\
k^{-} & =\frac{u\left(A_{2}\right)-u\left(A_{1}\right)}{\theta\left(A_{2}\right)-\theta\left(A_{1}\right)} \\
k^{+} & =\frac{u\left(A_{4}\right)-u\left(A_{3}\right)}{\theta\left(A_{4}\right)-\theta\left(A_{3}\right)}
\end{aligned}
$$

\subsection{P and D Parameters - Step Response}

After the static curve has been determined a step response is made to identify the linear process dynamics. Starting with the throttle in the limp-home position the control signal is ramped up until the position is slightly larger then $\theta_{l h}^{+}$. Then a step in control signal is applied and the position response is measured. The parameters in Equation (11) are fitted with the least mean squares method to the step response. A measured step response and the adapted model can be seen in Figure 9.

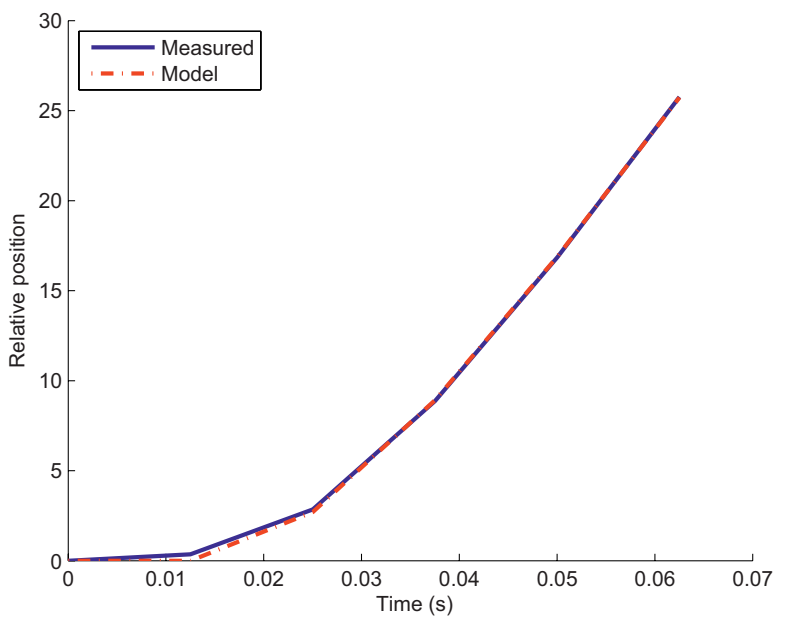

Figure 9

A measured step response together with simulation of the adapted process model. The initial position before the step has been set to zero. 


\subsubsection{How to Choose $\lambda$}

The tuning parameter $\lambda$ gives the rise time of the closed loop system. This can be translated into an arbitrary demand of the form "within $X \%$ in $t$ seconds" by considering the step response of the system in Equation (13) to a unit step.

$$
y(t)=1-e^{-t / \lambda}
$$

Setting $y(t)=1-X$ and solving for $\lambda$ gives:

$$
\lambda=\frac{-t}{\ln (X)}
$$

As was previously mentioned, this is only true when the control signal does not saturate. The increase in rise time due to signal saturation during large reference steps could be somewhat compensated for by decreasing $\lambda$. This will saturate the control signal for a longer period of time than with the original setting, thereby making up for lost time at the end of the step response, but is limited by the shortest possible rise time achieved with a saturated signal. Making $\lambda$ too small will make the PD-controller sensitive to measurement noise and make it use unnecessary large controller output.

\subsubsection{The $K_{p}$ and $K_{d}$ Parameters}

Once $\lambda$ is fixed, the relation between $K_{p}$ and $K_{d}$ is given by Equation (14c) from Section 2.4, restated here:

$$
\lambda=\frac{1+K_{d} K_{0}}{K_{p} K_{0}}
$$

Start by setting $K_{d}=0$ and do a reference step. Increase $K_{d}$ until an acceptable response is achieved, fine tuning can be done but is not necessary for the cases studied here. If $\lambda$ is too low, the desired rise time might not be achievable without overshoot. In that case $\lambda$ has to be increased and the process of selecting $K_{p}$ and $K_{d}$ restarted.

\subsubsection{The Filter Coefficient $\gamma$}

The filter coefficient in Equation (16) depend on the desired bandwidth of the filter and the sampling time of the controller. In the implementation $\gamma=0.7$ is used, which for a sampling time of $1 \mathrm{~ms}$ gives the filter a settling time (within $5 \%$ of end value) of less then $10 \mathrm{~ms}$. This has proved to work satisfactory in simulations and experiments.

\subsection{The I-Part}

As described in Section 2.5 the I-part of the controller is gain scheduled, using the tracking error to determine the integrator gain $K_{i}$. When $\left|e_{\theta}\right|$ is larger than $10 \%$, the integrator gain is small, $K_{i}=1$. As $\left|e_{\theta}\right|$ decreases from $10 \%$ to $1 \%, K_{i}$ increases linearly from 1 to 10 . From that point $K_{i}$ increases to 100 at $\left|e_{\theta}\right|=0.5 \%$ and remains constant when $\left|e_{\theta}\right| \leq 0.5 \%$, see Figure 10 .

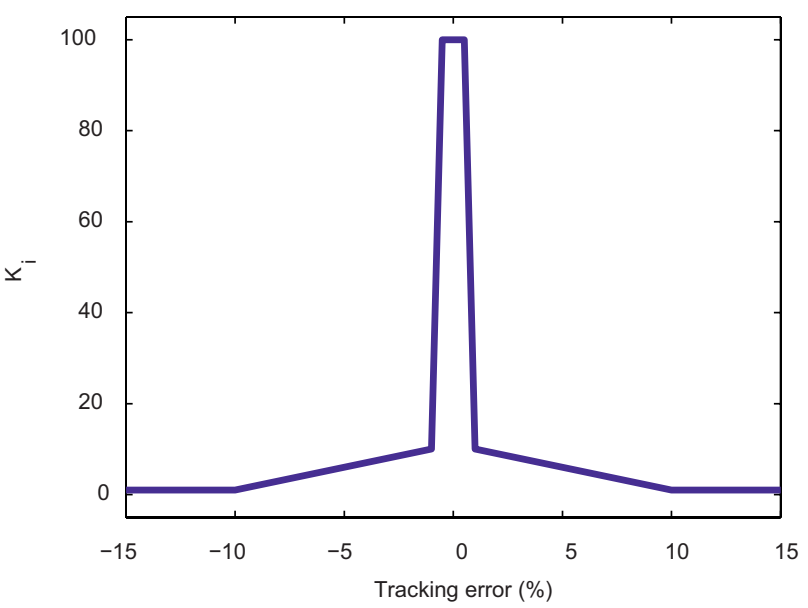

Figure 10

The integrator gain as a function of the tracking error. The large integrator gain close to zero tracking error help to quickly overcome modeling errors for small reference steps.

\section{SIMULATION RESULTS ON TC BENCHMARK MODEL}

The procedure described in Section 3 has been performed on the benchmark model provided by E-COSM'09, Zito et al. (2009), and resulted in the controller parameters in Table 1.

TABLE 1

Controller parameters identified with the two experiments on the throttle control benchmark model

\begin{tabular}{|l|l|l|l|l|l|}
\hline$K_{p}$ & 7.36 & $\theta_{l h}$ & 11.1 & $m_{l h}^{-}$ & -10.9 \\
$K_{d}$ & 0.03 & $\theta_{l h}^{+}$ & 11.3 & $k^{+}$ & 0.051 \\
$T_{c}^{+}$ & 8.76 & $\theta_{l h}^{-}$ & 10.9 & $k^{-}$ & 0.065 \\
$T_{c}^{-}$ & 6.83 & $m_{l h}^{+}$ & 9.03 & & \\
\hline
\end{tabular}

The controller performance is evaluated using the different reference signals provided by the benchmark model. These include a series of steps, ramps, and more arbitrary signals. Two different sized step responses are shown in Figure 11. For the large step the controller saturates the control signal until almost within $10 \%$ of the reference which indicates that the step response could not get much faster and the overshoot is less then $0.25 \%$. For the small step the throttle position is within the quantization error from the reference value in less than $20 \mathrm{~ms}$. The small chattering in the control signal originates from the PD-controller when the measurement oscillates rapidly around the measurement value. A ramp response (part of signal A2.2) and corresponding tracking error are shown in Figure 12. A small stick slip motion of the throttle is evident in the figure but the error does not exceed $0.3 \%$ during the ramp, which is 

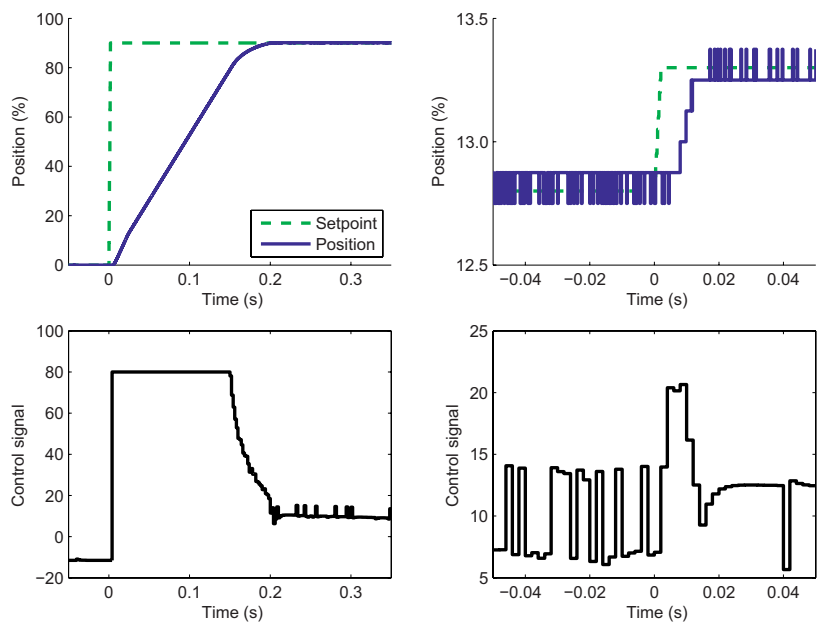

Figure 11

Two different sized step responses in $\theta_{\text {ref }}$ (part of signals A1.1 and A3.2) simulated on the benchmark model and the corresponding control signals. Settling time for the large step are less than $170 \mathrm{~ms}$ and the overshoot smaller than $0.25 \%$. The small step is within the quantization error from the reference in less than $20 \mathrm{~ms}$. A small chattering in the controls signal originating from the PD-controller occur when the measurement oscillates rapidly around the reference value.
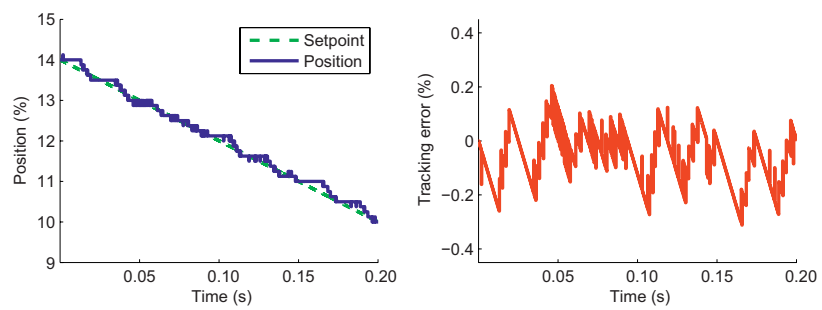

Figure 12

Simulated ramp response (the end of signal A2.2) and corresponding tracking error while passing through the limphome position, which is around $11.1 \%$. Peak tracking error is approximately $0.3 \%$.

small. All these results must be considered good and meet the demands on an automotive throttle controller.

The integral square error for all provided test signals in the throttle benchmark with the presented controller are given in Table 2. The initial throttle position was set equal to the initial reference value for each test.

\section{TABLE 2}

Performance measures for the tests defined in the TC benchmark (integral square error)

\begin{tabular}{|c|c|c|c|c|c|c|}
\hline A1.1 & A1.2 & A1.3 & A2.1 & A2.2 & A3.1 & A3.2 \\
\hline 835 & 150 & 20.0 & 0.17 & 0.13 & 1113 & 507 \\
\hline
\end{tabular}

\subsection{Robustness Investigation}

One critical parameter in the controller is the accuracy of the limp-home position. Due to the step-like characteristic of the static curve, incorrect information in the controller could lead to degraded controller performance around this position. Another important parameter is the amplitude of the friction compensator. During operation with only small changes in reference around a nominal value, a large part of the control signal comes from the friction compensator block. A bad estimate of the Coulomb friction could have large effect on the controller performance for small reference changes. To investigate the influence of incorrect limphome and friction compensation a series of test have been made.

\subsubsection{Error in Limp-Home Position}

Figures 13-16 show comparisons between the nominal controller and controllers that have an error in the limp-home compensator. In the figures to the left the limp-home compensator has the limp-home position at $11 \%$ which is the same as the actual position. In the figures to right the actual limp-home position is set to $13 \%$ in the model while the controller still believes it to be at $11 \%$.

In Figure 13 the controller starts to compensate for the limp-home position when the reference step to $11 \%$ is made, resulting in an overshoot. Small oscillations occur due to the stick slip motion of the throttle when the integral part have to overcome the limp-home torque to correct the position. In Figure 14 a ramp response through the limp-home position
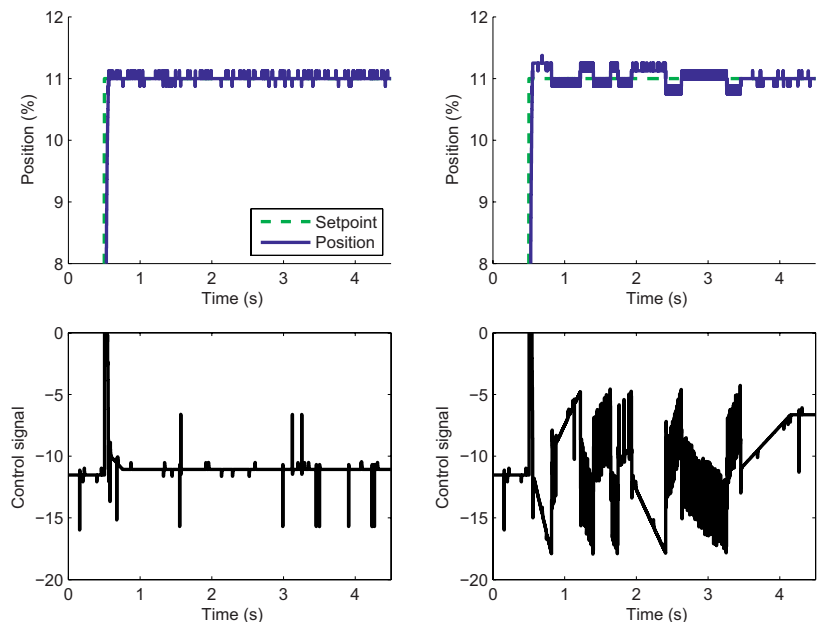

Figure 13

Left: the controller has correct information of the limp-home position. Right: the controller has a $2 \%$ error in limp-home position. The limp-home compensator starts to compensate below the limp-home position, resulting in an overshoot and small oscillations. 

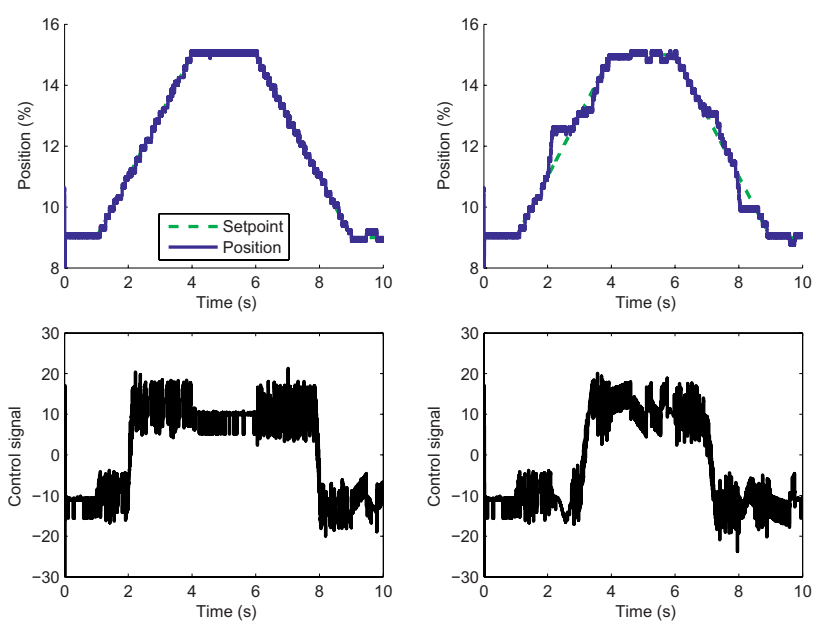

Figure 14

Left: the controller has correct information of the limp-home position. Right: the controller has a $2 \%$ error in limp-home position. When the reference passes the actual limp-home position (13\%) and where the controller believes the limphome position is $(11 \%)$, the tracking error rises to about $1 \%$ before the integrator compensates for the error.
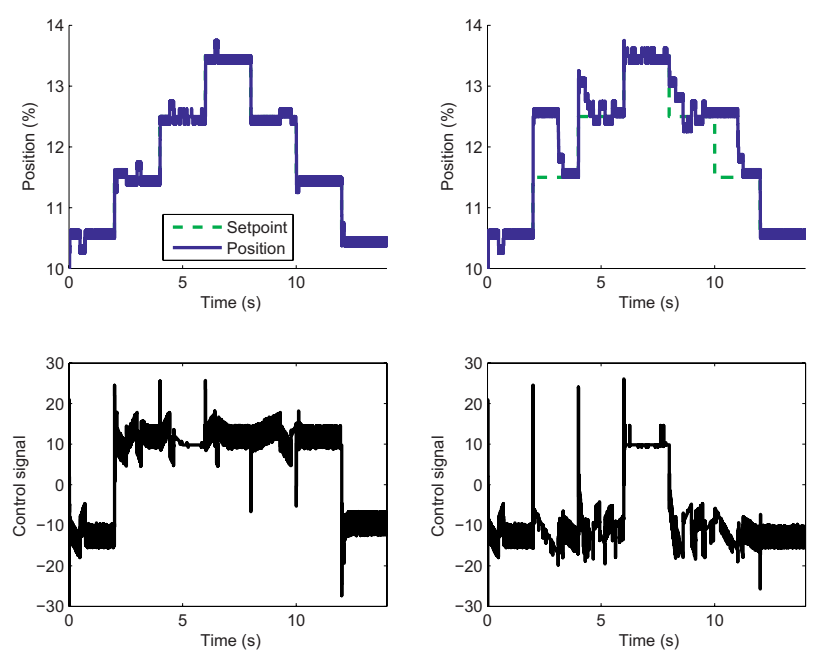

Figure 15

Left: the controller has correct information of the limp-home position. Right: the controller has a $2 \%$ error in limp-home position. The worst case scenario with limp-home position error. Small steps between the expected and actual limp-home position will cause over and undershoots of about $1 \%$.

is compared. For the controller with incorrect limp-home position the throttle position deviates slightly from the reference $(\leq 1.5 \%)$ both where the controller believes the limphome position is, and at the actual limp-home position.

Figure 15 shows step responses around the limp-home position with both the correct and the incorrect controller. The incorrect controller compensates for the limp-home
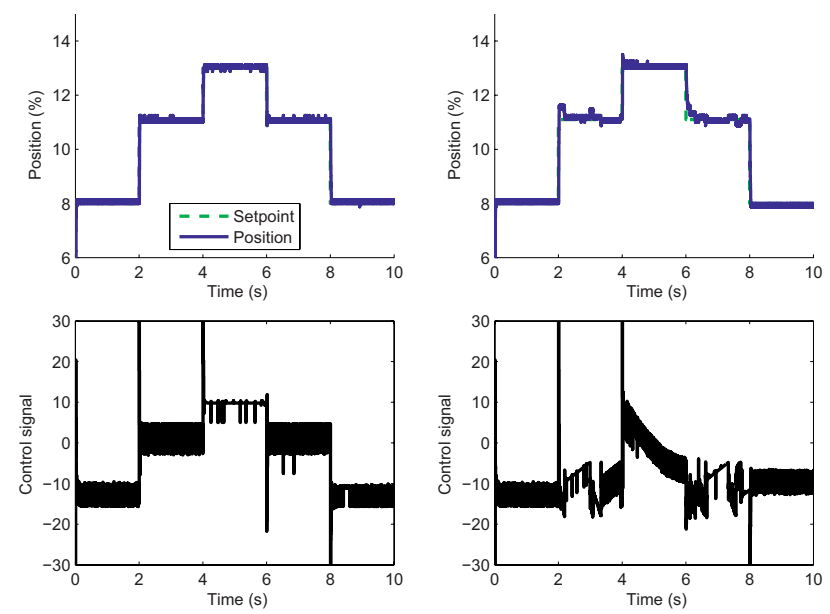

Figure 16

Left: the controller has correct information of the limp-home position. Right: the controller has a $2 \%$ error in limp-home position. The effect of the error in the controller is a small overshoot and undershoot.

position before it has been reached, leading to relatively large overshoot compared to the step size. In Figure 16 the behavior when doing steps into the limp-home position is shown. With an error in the controller, the position over- or undershoots of about $0.5 \%$.

An error in the limp-home compensator has a large effect on the controller performance when operating between the limp-home position in the controller and the actual limphome position. The largest impact is seen in Figure 15 and could lead to oscillations in the engine air flow that would result in oscillations in torque. This would be unacceptable in a production vehicle and thus this controller requires good precision in the parameter $\theta_{l h}$ in the limp-home compensator, within a few tenths of a percent.

The significance of this is further strengthened by experiments with real throttles, that have shown that there can be significant differences in the limp-home position between individual throttles. In particular a deviation larger than $2 \%$ has been found between an engine in an engine test cell and an identical engine in a vehicle. As a result of the incorrect calibration, torque variations could be felt by the driver in an operating point with a throttle reference in the proximity of the limp-home position. However, the desired accuracy is achieved with the presented tuning method. Effects like aging of the throttle and position sensor or production deviations could be handled by running a calibration at start up. If a full calibration is not possible, it is suggested that the system performs a simpler diagnosis and calibration by measuring the throttle position with zero control signal, which gives an accurate enough measurement of the limp-home position. 
To further improve the robustness of the controller it could be extended with on-line adaptation of the limp-home position and the static curve. This would amend problems that could arise due to parameter variations during a single engine run, for example due to variations in battery voltage and external temperature. These variations has been thoroughly discussed in Pavkvić et al. (2006) where an adaptive control is proposed, and is not treated here.

\subsubsection{Error in Friction Compensator}

Figure 17 compare the controller performance with correct friction estimation and where the friction has been underestimated of $30 \%$. Small oscillations around the reference value are introduced. An overestimated friction of $30 \%$ also tends to increase the position overshoot. These deviations are however fairly small and do not have a large effect on vehicle driveability. The controller is not very sensitive to friction compensator errors of this magnitude.
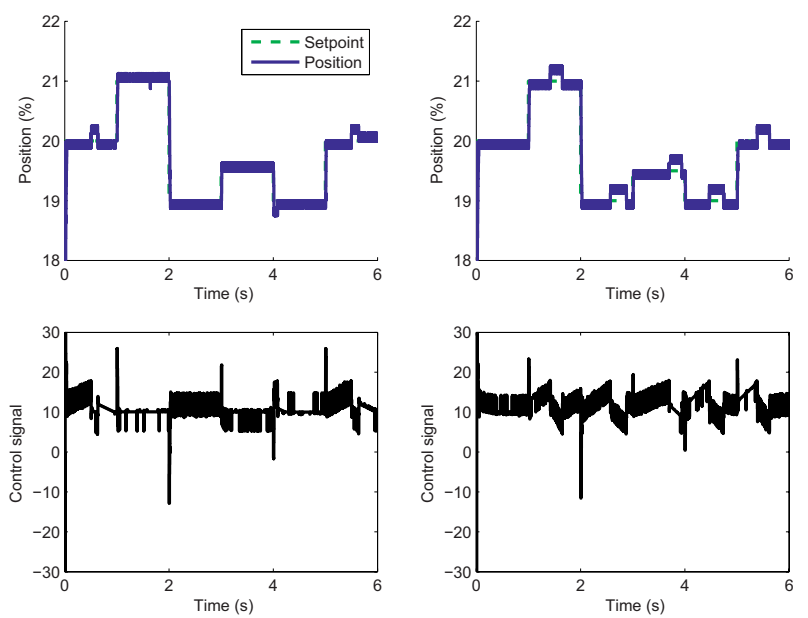

Figure 17

Left: the controller friction compensation is correct. Right: the friction compensator underestimates the friction of $30 \%$. Visible but small deviations from the reference value are introduced. The controller is not very sensitive to friction estimation errors of this magnitude.

\section{EXPERIMENTAL RESULTS}

The control design and tuning procedure have also been applied to the throttle in an engine test cell. The controller was evaluated using similar input signals as the benchmark model. A large and a small step response are shown in Figure 18, and a ramp with the corresponding tracking error are shown in Figure 19. The step response is slightly slower in the experimental tests but also has less overshoot. The control signal does not saturate during the step so the response time could be decreased by a lower value of $\lambda$.
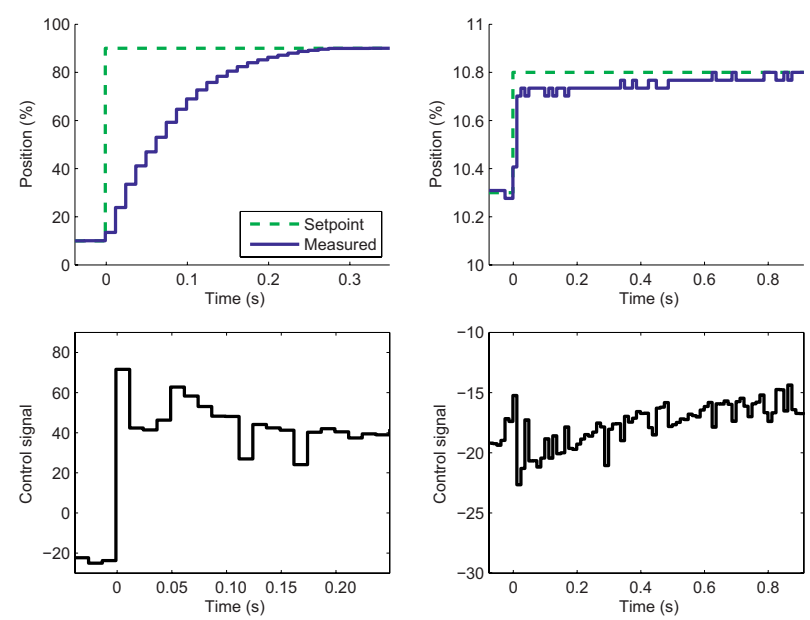

Figure 18

Two experimental step responses of different magnitude. The settling time for the large step is less than $200 \mathrm{~ms}$ and the overshoot is smaller than $0.1 \%$. The creeping effect seen in the small step could be explained by the higher measurement resolution for the experimental setup, approximately $0.033 \%$. The error after the initial step is less than $0.1 \%$.
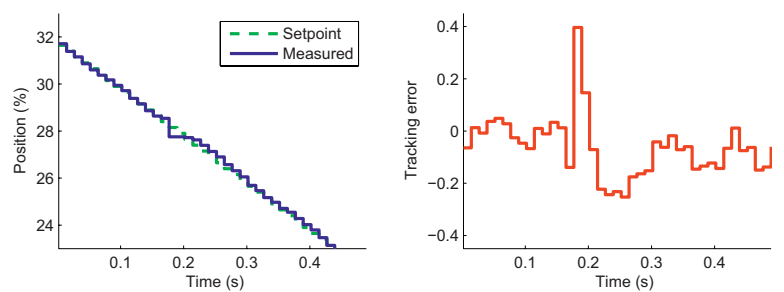

Figure 19

An experimental ramp response and corresponding tracking error which is slightly larger than on the simulation model. The error peaks at $0.4 \%$ when passing through the limp-home position but is otherwise below $0.2 \%$.

A creeping effect is visible for the small step, which could be explained by the higher measurement resolution, approximately $0.033 \%$, for this experimental setup compared to the TC benchmark model and standard production throttles. The error after the initial step is less than $0.1 \%$.

The tests show that the controller achieves satisfactory results also in experiments. These experimental results further strengthens the conclusion that the developed throttle controller gives good performance. The tuning method is straightforward to apply, and the controller performance is easy to tune with the aid of the tuning parameter $\lambda$ and the procedure described in Section 3.2.

\section{THE THROTTLE CONTROL BENCHMARK}

During E-COSM'09 the controller was evaluated together with five other participants, Vidal et al. (2009), Pozo et al. 
(2009), Colin and Chamaillard (2009), Reynoso-Meza et al. (2009) and one other contributor, during a series of tests in both SiL and HiL environment. The HiL evaluation showed that with the proposed calibration procedure, the controller met all specifications set by the benchmark in Zito et al. (2009). The results are presented in Zito and Tona (2009).

\section{CONCLUSIONS}

A throttle control strategy based on two static compensators and a PID controller has been presented. A tuning method for the parameters in both the compensator blocks and the PID controller has been developed. The relatively simple controller has been shown to give good performance both in simulation and in experiments. A robustness investigation has also been performed with respect to the friction and limp-home nonlinearities. An important result is that the controller is sensitive to how well the limp-home position is known. The accuracy of this controller parameter must be within a few tenths of a percent of the actual position in order to give a satisfactory control behavior in the neighborhood of the limp-home position. Experimental data have shown that deviations of a few percent between individual throttles can occur, which thus can pose a problem if this is not accounted for. The proposed design method and calibration procedure achieves a sufficiently accurate calibration. However, a simpler strategy for diagnosing and amending possible problems is to calibrate the limp-home position at each start up by registering and storing the throttle position with zero control signal.

\section{ACKNOWLEDGEMENTS}

This research was supported by the VINNOVA Industry Excellence Center LINK-SIC and by the Strategic Research Center MOVIII, funded by the Swedish Foundation for Strategic Research.

\section{REFERENCES}

Aström K.J., Hägglund T. (2006) Advanced PID Control, ISAThe Instrumentation, Systems, and Automation Society, 2006. ISBN 1-55617-942-1.

Colin G., Chamaillard Y. (2009) Pi and rst controllers for throttle actuator, in Proceedings of E-COSM'09, IFAC Workshop on Engine and Powertrain Control, Simulation and Modeling, IFP Rueil-Malmaison, France, 2009.
Deur J., Pavković D., Perić N., Jansz M., Hrovat D. (2004) An electronic throttle control strategy including compensation of friction and limp-home effects, IEEE Trans. Ind. Appl., 40, 821-834.

Eriksson L., Nielsen L. (2000) Non-linear model-based throttle control, in Electronic Engine Controls, SAE World Congress, Detroit, MI, USA, March 2000. Vol. SP-1500, pp. 47-51.

Olsson H., Aström K.J., Canudas de Wit C., Gäfvert M., Lischinsky P. (1998) Friction models and friction compensation, Eur. J. Control 4, 3, 176-195.

Pavkvić D., Deur J., Jansz M., Perić N. (2006) Adaptive control of automotive electronic throttle, Control Eng. Pract. 14, 121-136.

Pozo F., Acho L., Vidal Y. (2009) Nonlinear adaptive tracking control of an electronic throttle system: Benchmark experiments, in Proceedings of E-COSM'09, IFAC Workshop on Engine and Powertrain Control, Simulation and Modeling, IFP Rueil-Malmaison, France, 2009.

Reynoso-Meza G., Sanchis J., Blasco X. (2009) Multiobjective design of a digital controller for the throttle control benchmark, in Proceedings of E-COSM'09, IFAC Workshop on Engine and Powertrain Control, Simulation and Modeling, IFP RueilMalmaison, France, 2009.

Rivera D.E., Morari M., Skogestad S. (1986) Internal model control. 4. pid controller design, Ind. Eng. Chem. Process. Des. Dev. 25, 252-265.

Scattolini R., Siviero C., Mazzucco M., Ricci S., Poggio R., Rossi C. (1997) Modeling and identification of an electromechanical internal combustion engine throttle body, Control Eng. Pract. 5, 9, 1253-1259.

Vašak M., Baotić M., Morari M., Petrović I., Perić. N. (2006) Constrained optimal control of an electronic throttle, Int. J. Control 79, 465-478.

Vidal Y., Acho L., Pozo F. (2009) Robust control of an electronic throttle system via switched chattering control: Benchmark experiments, in Proceedings of E-COSM'09, IFAC Workshop on Engine and Powertrain Control, Simulation and Modeling, IFP Rueil-Malmaison, France, 2009.

Zito G., Tona P. (2009) The throttle control benchmark, Presentation at E-COSM'09, 2009, http://ecosm.nokiweb.fr/ images/TC $\backslash \% 20$ Benchmark.pdf.

Zito G., Tona P., Lassami P. (2009) The Throttle Control Benchmark, in Proceedings of E-COSM'09, IFAC Workshop on Engine and Powertrain Control, Simulation and Modeling, IFP Rueil-Malmaison, France, 2009.

Final manuscript received in April 2001 Published online in October 2011

\section{Copyright (C) 2011 IFP Energies nouvelles}

Permission to make digital or hard copies of part or all of this work for personal or classroom use is granted without fee provided that copies are not made or distributed for profit or commercial advantage and that copies bear this notice and the full citation on the first page. Copyrights for components of this work owned by others than IFP Energies nouvelles must be honored. Abstracting with credit is permitted. To copy otherwise, to republish, to post on servers, or to redistribute to lists, requires prior specific permission and/or a fee: Request permission from Information Mission, IFP Energies nouvelles, fax. +33147527096, or revueogst@ifpen.fr. 